
\title{
Research Sulure \\ Correlation of Brain Tumor-related Epilepsy With Clinicopathological Factors in Gliomas
}

\section{Bo Li}

Affiliated Hospital of Jining Medical University

\section{Shunli Jiang}

Affiliated Hospital of Jining Medical University

\section{Guangning Zhang}

Affiliated Hospital of Jining Medical University

Junchen Zhang ( $\boldsymbol{\sigma}$ junchenzhangjining@163.com )

Affiliated Hospital of Jining Medical University https://orcid.org/0000-0001-7258-6176

\section{Research Article}

Keywords: Glioma, Epilepsy, Neuropathology

Posted Date: May 26th, 2021

DOI: https://doi.org/10.21203/rs.3.rs-542204/v1

License: (c) (i) This work is licensed under a Creative Commons Attribution 4.0 International License. Read Full License 


\section{Abstract}

Objectives: Glioma patients with brain tumor-related epilepsy (BTRE) have a complex profile due to the simultaneous presence of two pathologies: glioma and epilepsy. However, the underlying pathophysiology of BTRE remains poorly understood. The purpose of this study is to investigate the correlation between molecular neuropathology and glioma with BTRE.

Methods: A retrospective cohort study of 186 glioma patients was evaluated at our hospital, with 64 presenting with BTRE. Chi-square test, spearman rank correlation and multivariate logistic analyses were used to identify clinicopathological factors associated with BTRE.

Results: Of the 186 patients examined in this study, 64 (34.4 \%) had BTRE. By analyzing the characteristics of these patients, the results showed that patient age (over 40 years; $p=0.007$ ), low WHO grade (grade I, II; $p=0.001$ ), IDH-1 positive mutation ( $p=0.027)$, ATR-X low expression level $(O R=0.44 ; 95 \%$ Cl: $0.21,0.92)$ and low Ki-67 proliferation index (OR=0.25; $95 \% \mathrm{Cl}: 0.10,0.68)$ were associated with the occurrence of BTRE. BTRE did not differ by sex, tumor location, expression of olig-2 or CD34. The results of the matching study showed that low Ki-67 proliferation index and negative ATR-X expression level were independent factors for a higher incidence of preoperative seizures in glioma patients.

Conclusion: The current study updates existing information on genetic markers in gliomas with BTRE and explores the correlation of a wide range of clinicopathological factors and glioma patients with BTRE. Our study suggests that three putative biomarkers for BTRE: positive IDH1 mutation, low Ki-67 proliferation index and negative ATR-X expression. These factors may provide insights for developing a more thorough understanding of the pathogenesis of epilepsy and effective treatment strategies aimed at seizure control.

\section{Introduction}

The epilepsy associated with brain tumors is called brain tumor-related epilepsy (BTRE). The International Union of Antiepileptics (ILAE) defines BTRE as a persistent tumor lesion in the brain that causes more than one seizure ${ }^{1-2}$. As the main symptom of brain tumors, epilepsy seriously affects the quality of life of patients. As a common brain tumor, most gliomas can cause epilepsy, especially in low-grade gliomas ${ }^{3}$. The frequency of epilepsy in patients with brain tumors ranges from 30 to $100 \%$ depending on tumor type ${ }^{1,3-5}$. Moreover, the proportion of epilepsy as the first symptom of gliomas can be as high as $50 \%-80 \%{ }^{6-7}$. Although surgical resection of the tumor can improve the prognosis of epilepsy, there are still a small number of patients after receiving surgical treatment can present with epilepsy difficultly controlled by drugs, known as refractory epilepsy ${ }^{8-9}$. As the most common presentations of glioma and one of the main clinical symptoms in the early stage of glioma, epileptic seizures are one of the most important reasons for many glioma patients to visit the clinic for the first time. Thus, seizures can also be considered as an early warning sign for glioma diagnosis ${ }^{10-13}$. Both epileptic seizure and the use of antiepileptic drugs can lead to cognitive impairment. Moreover, more than $10 \%$ of glioma patients will 
develop epileptic persistence, despite various antitumor treatments. One-half of the patients had a seizure within 1 month and two-thirds within 3 months before the last evaluation, affecting their long-term quality of life and the therapeutic effect of glioma ${ }^{14}$. Therefor, a better understanding of BTRE's associated risks and pathophysiological mechanisms can help to better predict, prevent and control the occurrence of epilepsy

Based on rprevious studies, the pathophysiological mechanisms of BTRE are numerous and complex. There is no consensus on the effect of histopathological types and biomarkers on epilepsy risk. The mechanism of BTRE is currently believed to be related to tumor mass effect ${ }^{15}$, formation of intracranial hypersensitized areas ${ }^{16}$, local microenvironment changes in surrounding tumor tissues ${ }^{17}$, and activation of glutamate NMDA receptor ${ }^{3}$. Some studies have found that patients had extremely excited epileptogenic foci in the brain, including tumor cells and surrounding adjacent tissues caused by tumor compression or stimulation, and they can disrupt the brain's balance between excitement and inhibition by altering the expression of neuropeptides, neurotransmitters and their receptors ${ }^{3,17}$. In addition, the regional destruction of nerve cells in tumor cells and adjacent tissues leads to the blocking of afferent nerves in some cortical areas, which promotes the formation of an epileptogenic environment and the gradual development of a highly sensitized area for epileptic seizures ${ }^{16}$. Other studies have shown that local microenvironmental changes in brain tumor, such as the swelling, ischemia, hypoxia and acidosis of the surrounding tissues of brain tumors, can also induce seizures. In addition, the depolarization and repolarization of ion channels on the cell membrane are unbalanced, so as to destroy the equilibrium of local microenvironment of $\mathrm{Na}^{+}, \mathrm{Ca}^{2+}$ and $\mathrm{Cl}^{-}$plasma, which increased the excitability of neurons, making them more vulnerable to external stimulation and thus, produce sudden, temporary abnormal discharge of neurons, leading to seizures. Studies have also shown that the NMDA receptor of glutamate can activate intracellular mTOR, AKT, MAPK and other signaling pathways, leading to an increase in the frequency of epileptic seizures and a faster growth rate of tumor cells ${ }^{3,18-19}$. Previous studies showed that the risk of seizures decreases with age and men were at higher risk than women. Tumor location (frontal, temporal, cap, pillow, island) and the WHO classification ( $\square \sim \bigotimes$ grade) were also associated with $\mathrm{BTRE}^{20-21}$. The incidence of BTREs in frontal lobe, temporal lobe, insular lobe and parietal lobe were higher than that in deep tumor tissues ${ }^{22}$.

Our study retrospectively analyzed the clinical and molecular pathologic data of all glioma patients in our hospital over a 3-year period, in order to study the relationship between gender, age, location of occurrence and different molecular pathological markers in the occurrence of brain tumor-related epilepsy in glioma patients, and we hope to have a more systematic and comprehensive understanding of epilepsy in glioma patients, and provide theoretical support for better improving the quality of life of glioma patients.

\section{Patients And Methods}

\section{Patients}


A total of 256 human glioma specimens diagnosed by pathology were collected from The Affiliated Hospital of Jining Medical University from January 2017 to December 2019, which were resected and preserved completely by neurosurgery. All cases had complete clinical, imaging and pathological data. MRI was performed in all patients after the hospitalization. Histological classifications were carried out according to the $2016 \mathrm{WHO}$ standards for grading tumors of the central nervous system. There were a total of $\varangle \sim \nabla$ level, and the histological types mainly included astrocytoma, oligodendroglioma, glioblastoma and etc.The diagnosis of epilepsy seizure is based on the criteria established by 2017 International League Against Epilepsy (ILAE), and semiological seizure classification was performed by preoperative evaluation. The exclusion criteria were as follows: (1) craniocerebral trauma; (2) congenital cerebral vascular malformation, such as arteriovenous malformation, cavernous hemangioma, etc; (3) other intracranial tumors, such as meningiomas, brain metastases, etc; (4) infectious diseases of the central nervous system; (5) primary epilepsy caused by congenital neurological diseases such as cortical dysplasia; (6) febrile epilepsy, drug-induced epilepsy and other epilepsy unrelated to intracranial tumors; (7) non-first operation, chemoradiotherapy and other treatments.

\section{Clinical variables}

The information was collected, including age, gender, epileptic seizure, lesion location, IDH-1 mutation, WHO classification, ki67 proliferation index, CD34 expression, GFAP expression, olig-2 expression and ATR-X expression (Table 1,2 for details). The diagnosis of epilepsy seizure was evaluated and guided jointly by certified neurologists and neurosurgeons. MRI was performed for all patients, and the lesion site was evaluated by a certified radiologist.

\section{Laboratory testing}

All glioma specimens were immunostained, including CD34, oligo-specific nuclear transcription factor (OLIg-2), ATRX, P53, proliferation index (KI-67), glial fibrillary acidic protein (GFAP). All of the above monoclonal antibodies were purchased from Beijing Zhongshan Jinqiao Biological Co. LTD, and sanger sequencing was used to detect IDH1 R132H mutation. Immunohistochemical (SP) method as follows: Paraffin sections were placed in the oven at $60^{\circ} \mathrm{C}$ for 1 hour to prevent defoliation, dewaxing, and rinsing with double steaming water for 5 minutes * 3 times; the slices were put into citric acid buffer solution with $\mathrm{pH}$ 6.0, put into pressure cooker and heated to boiling in water bath. After being kept for 2 minutes, the slices were naturally cooled at room temperature for 30 minutes, and rinsed with PBS for 5 minutes * 3 times. Add $3 \%$ hydrogen peroxide to inactivate endogenous peroxidase, leave it at room temperature for 5-10 minutes, and rinse it with distilled water for 5 minutes * 3 times. Add normal goat serum blocking solution, incubate at room temperature for 20 minutes, add 1:150 diluted primary antibody, and refrigerate overnight at $4{ }^{\circ} \mathrm{C}$. Rinse with PBS for $5 \mathrm{~min} * 3$ times, drip with biotin-labeled secondary antibody, leave at room temperature for 20 minutes, and rinse with PBS for 5 minutes * 3 times. Streptomycin antibiotic protein-horseradish catalase complex working solution was added, incubated at $37^{\circ} \mathrm{C}$ for $10-15$ minutes, and rinsed with PBS for 5 minutes * 4 times. DAB color developing agent was added to control the color developing under the microscope, tap water was fully rinsed, hematlignin was 
re-dyed for 25 seconds, $1 \%$ alcohol was differentiated for 50 seconds, ammonia returned to blue, tap water was fully rinsed, hematlignin was slightly re-dyed, dehydrated and transparent, neutral gum was used as the seal tablet, and PBS was used as the negative control instead of primary antibody. All operations were carried out in strict accordance with the kit instructions.

The YCTY2050 pathological image analysis system was used for quantitative determination. Specimens subjected to immunohistochemistry were assigned a grade for the staining intensity based on qualitative observations by two independent observers. GFAP was brownish yellow, stained by cytoplasm and cell membrane. ATRX, Ki67 and P53 were brownish yellow or brown particles, and the staining sites were nuclei. The staining sites of CD34 positive cells were mainly in the cell boundary, cytoplasm and uniform yellow staining of vascular endothelial cells. Olig-2 was brownish yellow, with nuclei and cytoplasm as the staining sites, mainly nuclei.

The immunostained sections were observed under low and high microscopes, and 5 visual fields were randomly selected under high microscopes (400X) to count 1,000 tumor cells and calculate the percentage of positive cells. The determination criteria for the results were as follows: negative $(-)$ : the tumor tissues were not stained at all or the number of positive cells was less than $5 \%$; weak positive (+) : the number of positive cells in tumor tissues was $6 \%-25 \%$; positive (++) : the number of positive cells in tumor tissues is $25 \%-50 \%$; strong positive (+++) : the positive number of tumor tissues is more than $50 \%$. For the convenience of statistics, weak positive and negative were classified as negative, while positive and strong positive were classified as positive. The Ki-67 proliferation index was defined as the percentage of immunoreactive tumor cell nuclei among the total number of cells. Proliferation index was evaluated as: the number of positive cells $\leq 5 \%$ was the low proliferation index; the number of positive cells $6-20 \%$ was the medium proliferation index; the number of positive cells $>20 \%$ was the high proliferation index.

\section{Statistical analyses}

The statistics were analyzed with SPSS, version 22.0 (SPSS Inc.,Chicago,IL,USA). Descriptive statistical methods were used to analyze the demographic characteristics, tumor location distribution and immunohistochemical indexes of the subjects. The frequency and percentage were used to describe the categorical variables statistically, and the Chi-Square test was used to analyze whether the difference between the two groups was statistically significant. Spearman rank correlation was used to analyze the correlation between immunohistochemical index and glioma with epilepsy. Multiple logistic regression was used to analyze the association between immunohistochemical indexes and the risk of glioma with epilepsy. The OR value and $95 \% \mathrm{Cl}$ were used as effect estimates. $\mathrm{P}<0.05$ was considered to be significant.

\section{Results}

\section{Descriptive characteristics of glioma patients}


256 patients were included initially. Of them, 226 patients were diagnosed with glioma after excluding cavernous hemangioma (15 cases), disordered cortical neurons (8 cases), glial hyperplasia (6 cases) and medulloblastoman (1 case). In addition, 40 patients were excluded due to unqualified specimens (insufficient tissue, damaged wax blocks). Finally, 186 patients were included (81 males and 105 females; age range, 4-73 years; median age, 50 years). We divided all patients into two groups based on preoperative seizure status, the BTRE group and the NO BTRE group. In the BTRE group, there were 64 patients, including 34 males and 30 females, $23(35.9 \%)$ of them were under the age 40 years and 41 $(64.1 \%)$ were over the age 40 years. In the NO BTRE group, there were 122 patients, including 47 males and 75 females, $22(18.0 \%)$ were under the age 40 years and 100 (82.0\%) were over the age 40 years. Tumor location was analyzed using MRI characteristics, and the tumor side was not involved in this study, due to better define the correlation between molecular indicators and tumor related with epilepsy. The most commonly involved lobe was the multiple $(n=65,34.9 \%)$, followed by the frontal $(n=55$, $29.6 \%)$, the temporal $(n=44,23.7 \%)$, the cerebellum $(n=7,3.8 \%)$, the parietal $(n=6,3.2 \%)$, the basal ganglia region $(n=3,1.6 \%)$, the thalamus and the occipital $(n=2,1.1 \%)$, the insula and the intraventricular $(n=1,0.5 \%)$ (for details see tables 1 and 2 ).

The pathological results showed that in the BTRE group 5 were grade I, 35 were grade II, 14 were grade III and 10 were grade IV; in the NO BTRE group, 7 were grade I, 32 were grade II, 40 were grade III and 43 were grade IV. The baseline information of all patients was summarized in Table 2. All the 186 patients were successfully collected and analyzed for the expression levels of GFAP, olig-2, ATR-X, CD34 and p53. IDH mutation and Ki67 proliferation index were also recorded and analyzed (Table 1). The histopathological type of all the patients were also collected and analyzed (2016 World Health Organization (WHO) Classification of Tumors of the Central Nervous System), see Table 2.

\section{Main results}

In order to identify factors that might be associated with tumor-related epilepsy, demographic and tumor information was compared between patients with seizures and those without (Table 1). Demographic statistics showed that female (56.5\%) glioma patients were significantly higher than male (43.5\%). However, male (53.1\%) had a higher incidence of BTRE than female (46.9\%). Chi-square test showed no statistical significance $(p=0.056)$. The statistical results showed that the glioma patients older than 40 years were significantly more than those younger than 40 years, and the incidence of tumor-related epilepsy in patients older than 40 years was significantly higher than that in patients younger than 40 years, with statistically significant differences $(p<0.05)$. The statistical results of correlation between tumor location and epilepsy showed that glioma patients tended to occur in the multiple lobes (34.9\%), followed by the frontal $(29.6 \%)$ and the temporal $(23.7 \%)$, while BTRE tended to occur in the frontal (35.9\%), followed by multiple (34.4\%) and temporal lobe (23.4\%), and the difference was not statistically significant $(\mathrm{p}=0.594)$. The WHO classification results showed that tumor-related epilepsy occurred mainly in patients with low-grade glioma (WHO grade II (54.7\%) ), but not common in patients with high-grade glioma WHO grade III (32.8\%), WHO grade IV (35.2\%), and the difference was statistically significant $(p<0.05)$. Histopathological results showed that glioblastoma IDH wild-type $(20.4 \%)$ and anaplastic 
astrocytoma IDH wild-type (17.7\%) were lower in the NO BTRE group, and diffuse astrocytoma IDH wildtype (23.4\%) was higher in the TRE group, and the difference of tumor histological type between the two groups was statistically significant $(p<0.001)$ (Table 2 ). Analysis of immunohistochemical indexes and IDH1 mutation results showed that three variables were significantly associated with the presence of epilepsy: IDH-1 mutation ( $p=0.027)$, ATR-X low expression level $(p=0.041)$, and low Ki-67 proliferation index $(p=0.001)$ (Table 2). Spearman rank correlation analysis showed that the correlation coefficient between IDH-1 mutation and BTRE was 0.16 , with a significant positive correlation $(p<0.05)$. The correlation coefficient between ATR-X positive expression and Ki-67 proliferation index and BTRE was -0.17 and -0.28 , respectively, and they were significantly negatively correlated with tumor-related epilepsy $(\mathrm{p}<0.05)$ (Table 3). Representative immu-nohistochemical staining for Ki-67 labeled with low to high (low, $\leq 5 \%$; medium, 6-20\%; high, $>20 \%$ ) and ATR-X positive or negative expression, and analysis of IDH1 mutation with negative and positive results, which shown in Figs. 1, 2 and 3, respectively. No other clinical or pathological variables were found to be associated with an increased risk for preoperative seizures in this group of patients. In the multinomial logistics regression analysis, after adjusting for gender, age, and WHO grade, the incidence of epilepsy in glioma patients with ATR-X positive expression was significantly reduced $(P=0.029)$ and the risk was $56 \%$ lower than in the ATR-X negative expression group ( $O R=0.44$; $95 \% \mathrm{Cl}: 0.21,0.92)$; Compared with the low-proliferation ki-67 group, the incidence of epilepsy in glioma patients in the high-proliferation $\mathrm{Ki}-67$ group was significantly reduced $(\mathrm{P}=0.006)$, and the risk was reduced by $75 \%(\mathrm{OR}=0.25 ; 95 \% \mathrm{Cl}: 0.10,0.68)$. That was to say, low Ki-67 proliferation index and low ATR$X$ expression level were found to have a strong association with preoperative seizures, which were independent factors for a higher incidence of preoperative seizures (Table 4).

\section{Discussion}

BTRE is a type of epilepsy, induced by the abnormal discharge of intracranial tumor itself or its mass effect caused by abnormal discharge of nerve cells around the lesion ${ }^{1}$. Seizures are one of the clinical symptoms of brain tumors, so patients can also present with disorders of consciousness, limb movement, sensory and autonomic nervous function ${ }^{23-25}$. Seizures are often seen in patients with brain tumors and occur at different rates. However, there is no clear conclusion on the pathogenesis and pathophysiology of BTRE, and, there is no single theory that can explain the cause of BTRE. Thus, the mechanism of BTRe is a relatively complex and multi-factor seizure ${ }^{26-29}$.

Seizures and the use of antiepileptic drugs can lead to cognitive impairment, affect the long-term quality of life of patients and the curative effect of brain tumor, which would also affect patients' family harmony, bad working condition, low moods ${ }^{27}$. Patients with BTRE present a complex therapeutic profile and require a unique and multidisciplinary approach. They, in fact, must face two different pathologies at the same time, brain tumor and epilepsy. In brain tumor patients, the presence of epilepsy is considered the most important risk factor for long-term disability ${ }^{30-31}$. The problem of the proper administration of medications and their potential side effects is of great importance, because good seizure control also has a significant impact on the patient's psychological and relational sphere ${ }^{31}$. Therefore, it is necessary to 
understand the mechanism of BTRE in order to better predict, prevent and control the occurrence of epilepsy. With the progress of research methods and technologies, BTRE has been deeply explored, especially in terms of its pathogenesis and the corresponding brain neural network. However, there is still a lack of sufficient attention and systematic research on the pathogenesis, clinical diagnosis and treatment of BTRE. At present, few studies have focused on the molecular neuropathologic correlation of glioma with tumor-related epilepsy ${ }^{32}$, beside several studies on the association between WHO classification, IDH1 mutation in BTRE of patients with low-grade glioma ${ }^{33-35}$. Therefore, in order to better define the etiology of tumor-related epilepsy in glioma patients, our study focused on the correlation between molecular neuropathic indicators and BTRE in low and high glioma patients, which was expected to clarify the pathogenesis of glioma patients with BTRE and benifit glioma patients.

To our knowledge, this is the first systematic retrospective study to investigate the correlation between molecular neuropathology and different grades glioma patients with BTRE. In the 186 glioma patients, the results showed that patients aged over 40 years old and low WHO grade were significantly correlated with BTRE. We identified one histopathological type and three molecular pathological characteristics that were specifically associated with BTRE: diffuse astrocytoma, IDH-wild type, low Ki-67 proliferation index, negative ATR-X expression and IDH-1 positive mutation status. Ki67 PI and ATR-X were independent correlation factors for a higher incidence of preoperative seizures. Future research should focus on identifying susceptibility candidate genes for BTRE in larger multicenter studies, including low- and highgrade gliomas with and without symptomatic seizures.

Previous studies have showed that age, sex, tumor location, histopathological type and WHO grade were the influencing factors of glioma with BTRE, although the results of various reports were inconsistent ${ }^{33,36-}$ 37. In our study, there is no agreement on the influence of age, sex and histopathological type on the occurrence of epilepsy. There was study showed that the risk was higher in men than in women, and the risk of seizure decreased with age. However, the results varied among different histopathological types ${ }^{38}$. Previous studies showed that the most common gliomas associated with epilepsy were diffuse astrocytoma, oligodendroglioma, and pleomorphic xanthoastrocytoma ${ }^{39}$. According to the WHO Classification, 2016 edition ${ }^{40}$, glioma are classified as diffuse gliomas (astrocytoma, oligodendroglioma, glioblastoma), as well as circumscribed and low-grade gliomas (angiocentric glioma, pilocytic astrocytoma, subependymal giant cell astrocytoma, pleomorphic xanthoastrocytoma, pilomyxoid astrocytoma, ependymoma, myxopapillary ependymoma, and subependymoma). Some study results showed that most patients with angiocentric glioma were children and young people, with no significant gender difference, and epilepsy was the main clinical manifestation ${ }^{41}$. Diffuse astrocytoma and oligodendroglial tumors were common in the cerebral hemisphere of young patients (frontal and temporal lobes were common), and epilepsy was one of the most common clinical symptoms ${ }^{42}$. These results showed that low-grade gliomas were more common in young adults, and were more common in supratentorial frontal and temporal lobes, with no gender difference and epilepsy was one of the main clinical symptoms. However, the previous studies were not entirely consistent with our findings. Our statistical results showed that BTRE was higher in male glioma patients than in female patients, but the 
results were not statistically significant. The incidence of BTRE in glioma patients older than 40 years was higher than that in glioma patients younger than 40 years, and the results were statistically significant. In addition, the most common histological types of BTRE in our study were diffuse astrocytoma, IDH-wildtype, followed by oligodendrogastrocytoma, NOS, Glioblastoma, IDH-wildtype and diffuse astrocytoma, IDH-mutant. The relationship between the location of the glioma and BTRE risk has been controversial. Gliomas can occur in any part of the brain. However, frontal, temporal, parietal gliomas are the most likely to be associated with BTRE, while occipital and supratentinal gliomas are less likely to have BTRE ${ }^{39}$. Our results showed that BTRE was most likely to occur in gliomas involving the frontal lobe and multiple lobes. Frontal lobe, temporal lobe, parietal lobe are closely related to human language, consciousness, and motor functions. Lesions in these areas could cause abnormal discharge of these brain functional areas, leading to epilepsy. If the glioma invades the cortical functional area, the incidence of epilepsy will be higher. However, statistical results showed that the occurrence of BTRE was not statistically significant in relation to the location of the tumor, which was different from previous studies $^{43}$. The reasons for the different statistical results may be related to differences in study design, the different populations and the complex mechanism of BTRE, and the results of individual research centers could be different.

Previous studies also showed that seizures were more common in low-grade gliomas than in high-grade gliomas $^{1,3}$. The results were similar to those in our study, which showed that BTRE was present in lower grade gliomas at $62.5 \%$ and in higher grade gliomas at $37.5 \%$. This may be related to the faster growth rate of high-grade glioma, which will cause severe ischemic and hypoxic damage to the surrounding tissues and tumor tissues themselves, making them less likely to form epileptogenic foci. Other studies had suggested that high grade gliomas release excessive amounts of the neuroexcitatory transmitter glutamate, which was neurotoxic and could lead to neuronal death in large quantities, so high-grade gliomas were less likely to have seizures ${ }^{44}$. Some studies also believed that the rapid growth of highgrade gliomas destroyed the brain's electrical transmission network. However, low-grade glioma had a slow growth rate and little influence on the surrounding tissues, presenting gradual degeneration, causing partial cortex afferent nerve block, leading to high sensitivity of demulsification, and promoting the formation of epileptogenic foci ${ }^{15,45}$. At present, the correlation and mechanism of BTRE in different WHO grades glioma mainly focus on the destruction of the blood-brain barrier, gene mutations, changes in neurotransmitters and receptors, and changes in ion concentration. In addition, some studies also showed that cellular inflammatory factors were involved in the occurrence and development of BTRE in glioma $^{46-47}$. Despite numerous studies, the mechanism of BTRE in different grades gliomas is still unclear and needed further research.

IDH is a member of the a-hydroxyacid oxidative decarboxylase family. IDH mainly acts on the tricarboxylic acid cycle to make isocitrate into a-ketoglutaric acid, while the IDH mutation could make isocitrate into 2-hydroxyglutaric acid (2-HG), and the altered reaction is also known as the weakening of alpha-ketoglutaric acid, which increases the level of $2-\mathrm{HG}^{48}$. The IDH mutation was first identified in lowgrade glioma patients, which was an early event in gliomagenesis and has significant implications for 
glioma progression and tumor behavior ${ }^{49}$. Previous study showed that mutation of the IDH gene occurred in $~ 80 \%$ of lower-grade (WHO grade II and grade III) gliomas ${ }^{50}$. Mutant IDH produces (R)-2hydroxyglutarate, which induces DNA hypermethylation and presumably drives tumorigenesis, also suggests that 2-HG plays an important role in the occurrence and development of epilepsy. Due to 2-HG structurally similar to glutamate, IDH1 mutant glioma cells release a large number of 2-HG will combine multiple neurons and activate receptors, such as NMDAR and AMPAR, causing the influx of sodium ion, potassium ion and calcium ion, and increasing the action potential triggered by neurons, then damaging the balance between inhibition and excitation of neurons, thus causing abnormal excitation of neurons and promoting the occurrence of epilepsy ${ }^{51}$. Therefore, the incidence of BTRE in IDH1 mutant glioma patients is much higher than that in IDH1 wild-type patients. Our study showed that the IDH1 positive mutation rate of glioma patients with epilepsy was $23.4 \%$, which was different from IDH1 negative mutation in NO BTRE group (13.9\%), and the difference was statistically significant, which suggested that IDH1 positive mutation was closely related to BTRE in glioma patients. Moreover, our study also showed that IDH1 mutation was more likely to occur in diffuse astrocytoma patients.

In our study, we have identified two important molecular pathological characteristics associations with BTRE in glioma patient, which were $\mathrm{Ki}-67 \mathrm{PI}$ and ATR-X expression. Tumor-correlated biomarkers in glioma patient with BTRE have been a popular research topic for decades, however, they have not traditionally enjoyed as much attention as more malignant brain tumor. In recent years, a number of developments have been achieved towards further understanding of the molecular and developmental backgrounds of glioma patient with BTRE, which helps to clarify the mechanism of glioma leading to epileptic seizures. There were several studies showed that a number of candidate genes that cause seizures in glioma patients, like the interleukin-1 $\beta$ (IL-1 $\beta)^{52}, \mathrm{CD} 34^{53}$, tuberous sclerosis complex (TSC) ${ }^{54}$ and et al. Molecular genetic effects may alter the balance between intracortical inhibitory and excitatory mechanisms, therefore inducing epileptogenic activity. However, the molecular mechanism underlying the BTRE remain largely unknown, and there still have issue on whether BTRE in glioma patients results from localized epileptic foci that lead to abnormal circuits or from molecular defects. Ki- 67 was a protein found in the nucleus of a cell. Ki was discovered in the 1980s in Kiel, Germany, and the number 67 comes from the experiment number. The researchers found a strong link between the amount of this protein and the cell's division cycle; the higher the positive rate of ki67, the higher the proportion of cells in the proliferative cycle and the faster the tumor growth ${ }^{55}$. At present, ki67 has become a very important indicator to judge the activity level of tumor cells. The association between ki67 and BTRE in glioma patients has been rarely studied and the results were inconsistent ${ }^{56}$. One study showed that the ki67 proliferation index was not associated with the risk of epileptic seizures in glioma patients ${ }^{57}$. However, some studies have also proved that ki-67 significantly affected seizure prognosis ${ }^{58}$, and related with BTRE in glioma by increasing proliferation ${ }^{59}$. These results indicated that ki67 may play a role in epilepsy in glioma patients. As we know, Low ki-67 PI is usually associated with benign tumors or low grade glioma(WHO grade I, II), and our study already showed that WHO grade I or II was associated with BTRE in glioma patients(table 2), which supports our hypothesis that it could increased incidence of 
preoperative seizures in glioma patients. In our study, low ki67 proliferation index was clearly associated with BTRE in glioma, with statistical significance. Of course, the results of this study cannot be taken as the final result, and the correlation between ki67 proliferation index and epilepsy in glioma patients needs further study. ATR-X gene is a pathogenic gene of a thalassemia/mental retardation syndrome X-linked located on the $\mathrm{X}$ chromosome, and plays an important role in chromatin remodeling, genome and maintenance of telomere stability ${ }^{60}$. Mutations in ATR-X have been identified in multiple tumor types. In 2011, Heaphy et al. found that ATR-X gene mutation existed in tumors of the central nervous system, and a series of subsequent studies confirmed that ATR-X gene mutation mainly occurred in diffuse astrocytoma $^{61}$; In 2014, the consensus of the International Society of Neuropathology considered the deletion of ATR-X gene as a characteristic molecular marker of diffuse astrocytoma ${ }^{62}$. In 2016 , the WHO integrated IDH, TERT, 1P19Q and other molecular markers into the histopathological diagnosis of glioma, promoting the accurate diagnosis of glioma ${ }^{40}$. Under the guidance of molecular typing, accurate treatment of glioma has been a hot research topic in recent years. ATR-X gene mutation can be used as a prognostic indicator for glioma patients. However, the association between ATR-X and epilepsy in glioma patients has not been described. Our study showed that compared with the epilepsy group, glioma patients with ATR-X gene mutation in the non-epileptic group were less likely to develop epilepsy, which was statistically significant. In this study, positive expression of ATR-X gene in glioma patients was significantly negatively correlated with tumor concurrent epilepsy $(p<0.05)$ \which was shown to be an independent predictor for preoperative seizures in glioma patients, a finding that has not been previously reported, and the mechanism needs to be confirmed by further research. Therefore, we speculate that Ki67 and ATR-X may become the novel diagnostic or therapeutic targets for tumor-related epilepsy in glioma in the future.

In our study, we did not find that glioma patients with tumors of different lobes, positive or negative expression of olig-2 and CD34 were significantly likely to present with epilepsy. These results differ from previous studies and may be due to differences in the number of cases, methods, and mechanisms involved $^{53}$. In addition, some limitations should be presented as follows. First, the project design of small sample $(\mathrm{N}=186)$ and single center cannot produce effective results. Second, there were only 64 glioma patients had epilepsy, leading to a relatively limited power. Thus, the adjustment in logistic regression may be too extensive. Third, there were no data for other molecular, like p53, EGFR amplification, 1p/19q deletion, which should be clarified their correlation with BTRE in glioma patients. Thus, further studies are needed to provide more evidence to identify molecular markers related in glioma with BTRE.

In summary, this cohort study was performed new molecular pathologic indicators in glioma patients with BTRE. Low WHO grade, glioma patients over 40 years old, IDH1 positive mutation, as well as low ki67 proliferation index and ATR-X negative expression can predict the likelihood of epilepsy occurring in glioma patients. Thus, some of these factors will be worthy of further research as a possible therapeutic target, which may provide insights into developing a more thorough understanding of the pathogenesis of epilepsy and aim to improve the long-term remission rate of epilepsy before and after surgery. 


\section{Declarations}

\section{ETHICAL STATEMENT}

\section{Ethics approval and consent to participate}

The study was approved by the Institute's Ethics Committee of Affiliated Hospital of Jining Medical University, Jining, China. The patient's consent and informed consent were obtained before the collection of tissue samples from the patient.

\section{Consent for publication}

The patients in this study agreed to publication of this manuscript.

\section{Availability of Data and Materials}

All data generated or analyzed during this study are included in this published article.

\section{Conflics of interest}

The authors declare that they have no competing interests.

\section{Funding}

This work was supported by the PhD Research Foundation of Affiliated Hospital of Jining Medical University (2021-BS-006).

\section{Authors' contributions}

Conception and design: Bo Li and Junchen Zhang; Collection and assembly of data: Bo Li, Shunli Jiang, Guangning Zhang, and Junchen Zhang: Data analysis and interpretation: Bo Li, Shunli Jiang2, Guangning Zhang, and Junchen Zhang: Manuscript writing: Bo Li and Junchen Zhang: Final approval of manuscript: All authors read and approved the final manuscript.

\section{Acknowledgements}

Thanks so much to my teachers Shuo Wang, Shugan Zhu and Andrew kaye for encouraging me to finish the work.

\section{References}

1. Pallud J, McKhann GM (2019) Diffuse Low-Grade Glioma-Related Epilepsy. Neurosurg Clin N Am 30(1):43-54

2. Wessling C, Bartels S, Sassen R, Schoene-Bake JC, von Lehe M (2015) Brain tumors in children with refractory seizures-a long-term follow-up study after epilepsy surgery. Childs Nerv Syst 31(9):1471- 
3. van Breemen MS, Wilms EB, Vecht CJ (2007) Epilepsy in patients with brain tumours: epidemiology, mechanisms, and management. Lancet Neurol 6(5):421-430

4. Brogna C, Gil Robles S, Duffau H (2008) Brain tumors and epilepsy. Expert Rev Neurother 8(6):941955

5. Avila EK, Chamberlain M, Schiff D et al. Seizure control as a new metric in assessing efficacy of tumor treatment in low-grade glioma trials [published correction appears in Neuro Oncol. 2017 Feb 1;19(2):304]. Neuro Oncol. 2017;19(1):12-21

6. Kerkhof M, Vecht CJ (2013) Seizure characteristics and prognostic factors of gliomas. Epilepsia 54(Suppl 9):12-17

7. Hildebrand J, Lecaille C, Perennes J, Delattre JY (2005) Epileptic seizures during follow-up of patients treated for primary brain tumors. Neurology 65(2):212-215

8. You G, Sha Z, Jiang T (2012) The pathogenesis of tumor-related epilepsy and its implications for clinical treatment. Seizure 21(3):153-159

9. Berntsson SG, Malmer B, Bondy ML, Qu M, Smits A (2009) Tumor-associated epilepsy and glioma: are there common genetic pathways? Acta Oncol 48(7):955-963

10. Santos-Pinheiro F, Park M, Liu D et al (2019) Seizure burden pre- and postresection of low-grade gliomas as a predictor of tumor progression in low-grade gliomas. Neurooncol Pract 6(3):209-217

11. Wasilewski A, Serventi J, Ibegbu C, Wychowski T, Burke J, Mohile N (2020) Epilepsy education in gliomas: engaging patients and caregivers to improve care. Support Care Cancer 28(3):1405-1409

12. You G, Yan W, Zhang W et al (2012) Significance of miR-196b in tumor-related epilepsy of patients with gliomas. PLoS One 7(9):e46218

13. Gallagher P, Leach JP, Grant R (2014) Time to focus on brain tumor-related epilepsy trials. Neurooncol Pract 1(3):123-133

14. Hildebrand J, Lecaille C, Perennes J, Delattre JY (2005) Epileptic seizures during follow-up of patients treated for primary brain tumors. Neurology 65(2):212-215

15. Wolf HK, Roos D, Blümcke I, Pietsch T, Wiestler OD (1996) Perilesional neurochemical changes in focal epilepsies. Acta Neuropathol 91(4):376-384

16. Terrone G, Salamone A, Vezzani A (2017) Inflammation and Epilepsy: Preclinical Findings and Potential Clinical Translation. Curr Pharm Des 23(37):5569-5576

17. Shamji MF, Fric-Shamji EC, Benoit BG (2009) Brain tumors and epilepsy: pathophysiology of peritumoral changes. Neurosurg Rev 32(3):275-286

18. Lai MC, Tzeng RC, Huang CW, Wu SN (2019) The Novel Direct Modulatory Effects of Perampanel, an Antagonist of AMPA Receptors, on Voltage-Gated Sodium and M-type Potassium Currents. Biomolecules 9(10):638

19. Yuan $Y$, Xiang W, Yanhui $L$ et al (2017) Activation of the mTOR signaling pathway in peritumoral tissues can cause glioma-associated seizures. Neurol Sci 38(1):61-66 
20. Adamek D, Siwek GP, Chrobak AA et al (2016) Angiocentric glioma from a perspective of A-B-C classification of epilepsy associated tumors. Folia Neuropathol 54(1):40-49

21. Englot DJ, Berger MS, Chang EF, Garcia PA (2012) Characteristics and treatment of seizures in patients with high-grade glioma: a review. Neurosurg Clin N Am 23(2):227-227viii

22. Yu Z, Zhang N, Hameed NUF et al (2019) The Analysis of Risk Factors and Survival Outcome for Chinese Patients with Epilepsy with High-Grade Glioma. World Neurosurg 125:e947-e957

23. Goldstein ED, Feyissa AM (2018) Brain tumor related-epilepsy. Neurol Neurochir Pol 52(4):436-447

24. Maschio M, Zarabla A, Maialetti A et al (2017) Quality of life, mood and seizure control in patients with brain tumor related epilepsy treated with lacosamide as add-on therapy: A prospective explorative study with a historical control group. Epilepsy Behav 73:83-89

25. Englot DJ, Chang EF, Vecht CJ (2016) Epilepsy and brain tumors. Handb Clin Neurol 134:267-285

26. Vercueil L (2011) Brain tumor epilepsy: a reappraisal and six remaining issues to be debated. Rev Neurol (Paris) 167(10):751-761

27. Armstrong TS, Grant R, Gilbert MR, Lee JW, Norden AD (2016) Epilepsy in glioma patients: mechanisms, management, and impact of anticonvulsant therapy. Neuro Oncol 18(6):779-789

28. Vecht C, Royer-Perron L, Houillier C, Huberfeld G (2017) Seizures and Anticonvulsants in Brain Tumours: Frequency, Mechanisms and Anti-Epileptic Management. Curr Pharm Des 23(42):64646487

29. Roberts M, Northmore T, Shires J, Taylor P, Hayhurst C (2018) Diffuse low grade glioma after the 2016 WHO update, seizure characteristics, imaging correlates and outcomes. Clin Neurol Neurosurg 175:9-15

30. Vecht CJ, Wilms EB (2010) Seizures in low- and high-grade gliomas: current management and future outlook. Expert Rev Anticancer Ther 10(5):663-669

31. Politsky JM (2017) Brain Tumor-Related Epilepsy: a Current Review of the Etiologic Basis and Diagnostic and Treatment Approaches. Curr Neurol Neurosci Rep 17(9):70

32. You G, Sha Z, Jiang $T$ (2012) The pathogenesis of tumor-related epilepsy and its implications for clinical treatment. Seizure 21(3):153-159

33. Goldstein ED, Feyissa AM (2018) Brain tumor related-epilepsy. Neurol Neurochir Pol 52(4):436-447

34. Phan K, Ng W, Lu VM et al (2018) Association Between IDH1 and IDH2 Mutations and Preoperative Seizures in Patients with Low-Grade Versus High-Grade Glioma: A Systematic Review and MetaAnalysis. World Neurosurg 111:e539-e545

35. Wang ZF, Chen HL (2016) Relationship between IDH1 mutation and preoperative seizure in low-grade gliomas: A meta-analysis. Clin Neurol Neurosurg 148:79-84

36. Chang EF, Potts MB, Keles GE et al (2008) Seizure characteristics and control following resection in 332 patients with low-grade gliomas. J Neurosurg 108(2):227-235

37. Suzuki H, Mikuni N, Sugita S et al (2020) Molecular Aberrations Associated with Seizure Control in Diffuse Astrocytic and Oligodendroglial Tumors. Neurol Med Chir (Tokyo) 60(3):147-155 
38. GBD 2016 Epilepsy Collaborators. Global, regional, and national burden of epilepsy, 1990-2016: a systematic analysis for the Global Burden of Disease Study 2016 [published correction appears in Lancet Neurol (2019 May;18(5):e4]) Lancet Neurol. 2019;18(4):357-375

39. Chen DY, Chen CC, Crawford JR, Wang SG (2018) Tumor-related epilepsy: epidemiology, pathogenesis and management. J Neurooncol 139(1):13-21

40. Louis DN, Perry A, Reifenberger G et al (2016) The 2016 World Health Organization Classification of Tumors of the Central Nervous System: a summary. Acta Neuropathol 131(6):803-820

41. Adamek D, Siwek GP, Chrobak AA et al (2016) Angiocentric glioma from a perspective of A-B-C classification of epilepsy associated tumors. Folia Neuropathol 54(1):40-49

42. Weller M, van den Bent M, Tonn JC et al (2017 Nov;18(11):e642]) European Association for NeuroOncology (EANO) guideline on the diagnosis and treatment of adult astrocytic and oligodendroglial gliomas [published correction appears in Lancet Oncol. Lancet Oncol. 2017;18(6):e315-e329

43. Chang EF, Potts MB, Keles GE et al (2008) Seizure characteristics and control following resection in 332 patients with low-grade gliomas. J Neurosurg 108(2):227-235

44. Sørensen MF, Heimisdóttir SB, Sørensen MD et al (2018) High expression of cystine-glutamate antiporter $\mathrm{XCT}$ (SLC7A11) is an independent biomarker for epileptic seizures at diagnosis in glioma. $J$ Neurooncol 138(1):49-53

45. Norden AD, Blumenfeld H (2002) The role of subcortical structures in human epilepsy. Epilepsy Behav 3(3):219-231

46. Aronica E, Ravizza T, Zurolo E, Vezzani A (2012) Astrocyte immune responses in epilepsy. Glia 60(8):1258-1268

47. Vezzani A, Lang B, Aronica E (2015) Immunity and Inflammation in Epilepsy. Cold Spring Harb Perspect Med 6(2):a022699. Published 2015 Dec 18

48. Maus A, Peters GJ (2017 Jun;49(6):1143]) Glutamate and a-ketoglutarate: key players in glioma metabolism [published correction appears in Amino Acids. Amino Acids. 2017;49(1):21-32

49. Watanabe T, Nobusawa S, Kleihues P, Ohgaki H (2009) IDH1 mutations are early events in the development of astrocytomas and oligodendrogliomas. Am J Pathol 174(4):1149-1153

50. Stockhammer F, Misch M, Helms HJ et al (2012) IDH1/2 mutations in WHO grade II astrocytomas associated with localization and seizure as the initial symptom. Seizure 21(3):194-197

51. Chen H, Judkins J, Thomas C et al (2017) Mutant IDH1 and seizures in patients with glioma. Neurology 88(19):1805-1813

52. Heida JG, Pittman QJ (2005) Causal links between brain cytokines and experimental febrile convulsions in the rat. Epilepsia 46(12):1906-1913

53. Giulioni M, Marucci G, Cossu M et al (2019) CD34 Expression in Low-Grade Epilepsy-Associated Tumors: Relationships with Clinicopathologic Features. World Neurosurg 121:e761-e768

54. van Slegtenhorst $M$, Nellist $M$, Nagelkerken B et al (1998) Interaction between hamartin and tuberin, the TSC1 and TSC2 gene products. Hum Mol Genet 7(6):1053-1057 
55. Scholzen T, Gerdes J (2000) The Ki-67 protein: from the known and the unknown. J Cell Physiol 182(3):311-322

56. Yang P, You G, Zhang W et al (2014) Correlation of preoperative seizures with clinicopathological factors and prognosis in anaplastic gliomas: a report of 198 patients from China. Seizure 23(10):844-851

57. Toledo M, Sarria-Estrada S, Quintana M et al (2017) Epileptic features and survival in glioblastomas presenting with seizures. Epilepsy Res 130:1-6

58. Yuan Y, Xiang W, Yanhui L et al (2013) Ki-67 overexpression in WHO grade II gliomas is associated with poor postoperative seizure control. Seizure 22(10):877-881

59. Yuan Y, Xiang W, Yanhui L et al (2013) Ki-67 overexpression in WHO grade II gliomas is associated with poor postoperative seizure control. Seizure 22(10):877-881

60. Schenkel LC, Kernohan KD, McBride A et al (2017) Identification of epigenetic signature associated with alpha thalassemia/mental retardation X-linked syndrome. Epigenetics Chromatin 10:10

61. Heaphy CM, de Wilde RF, Jiao Y et al (2011) Altered telomeres in tumors with ATRX and DAXX mutations. Science 333(6041):425

62. Haberler C, Wöhrer A (2014) Clinical Neuropathology practice news 2-2014: ATRX, a new candidate biomarker in gliomas. Clin Neuropathol 33(2):108-111

\section{Tables}


Table 1. Basic characteristics and immunohistochemical indexes of the study population.

$\begin{array}{lllll}\text { Characteristics } & \begin{array}{l}\text { Total } \\ \text { cases }\end{array} & \begin{array}{l}\text { Cases with epilepsy } \\ (\mathrm{N}=64)\end{array} & \begin{array}{l}\text { Cases without epilepsy } \\ (\mathrm{N}=122)\end{array} & \begin{array}{c}P \text { - } \\ \text { value }\end{array} \\ & & & \\ & & \end{array}$

Sex, N (\%)

0.056

$\begin{array}{llll}\text { Male } & 81(43.5) & 34(53.1) & 47(38.5) \\ \text { Female } & 105(56.5) & 30(46.9) & 75(61.5)\end{array}$

Age, years, N

$(\%)$

$\begin{array}{llll}<40 & 45(24.2) & 23(35.9) & 22(18.0) \\ \geq 40 & 141(75.8) & 41(64.1) & 100(82.0)\end{array}$

Immunohistochemical

indexes

GFAP, N (\%)

0.530

$\begin{array}{llll}(-) & 2(1.1) & 0(0.0) & 2(1.6) \\ (+) & 182(97.8) & 63(98.4) & 119(97.5) \\ \text { Missing } & 2(1.1) & 1(1.6) & 1(0.8)\end{array}$

Olig-2, N (\%)

0.942

$\begin{array}{llll}(-) & 7(3.8) & 2(3.1) & 5(4.1) \\ (+) & 167(89.8) & 58(90.6) & 109(89.3) \\ \text { Missing } & 12(6.5) & 4(6.3) & 8(6.6)\end{array}$

IDH-1, N (\%)

0.027

$\begin{array}{lllll}(-) & 133(71.5) & 38(59.4) & 95(77.9) & \\ (+) & 32(17.2) & 15(23.4) & 17(13.9) & 0.041 \\ \text { Missing } & 21(11.3) & 11(17.2) & 10(8.2) & \\ \text { ATR-X, N (\%) } & & & 41(33.6) & \\ (-) & 70(37.6) & 29(45.3) & 69(56.6) & 0.001 \\ (+) & 93(50.0) & 24(37.5) & 12(9.8) & \end{array}$




\begin{tabular}{|c|c|c|c|c|}
\hline Medium & $73(39.2)$ & $28(43.8)$ & 45 (36.9) & \\
\hline High & $72(38.7)$ & $14(21.9)$ & $58(47.5)$ & \\
\hline \multicolumn{5}{|c|}{ CD34, N (\%) } \\
\hline$(-)$ & $30(16.1)$ & $9(14.1)$ & $21(17.2)$ & 0.731 \\
\hline$(+)$ & $123(66.1)$ & $42(65.6)$ & $81(66.4)$ & \\
\hline Missing & $33(17.7)$ & $13(20.3)$ & $20(16.4)$ & \\
\hline
\end{tabular}

Of the 186 glioma patients, 56.5 percent were women and 75.8 percent were aged 40 or older. In terms of immunohistochemical indexes, the positive rate of IDH-1 in the epileptic group was significantly higher than that in the non-epileptic group, but the positive rate of ATR-X and ki-67 proliferation were significantly lower than that in the non-epileptic group (all $\mathrm{P}<0.05$ ). 
Table 2. Pathological grade, tumor location, and histopathological type of the study population.

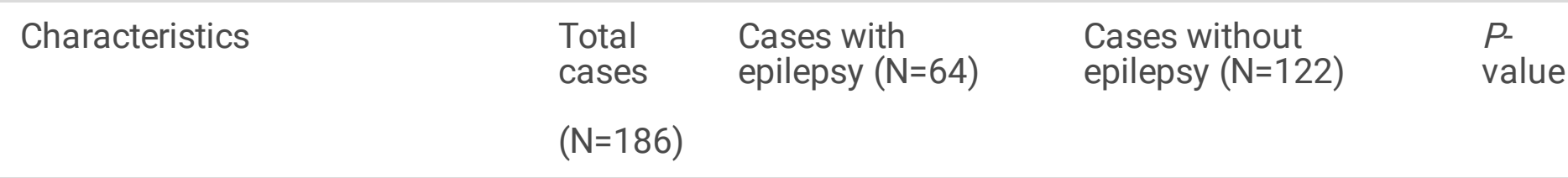

Pathological grade, N (\%)

0.001

$\begin{array}{llll}\text { Grade } 1 & 12(6.5) & 5(7.8) & 7(5.7) \\ \text { Grade 2 } & 67 & 35(54.7) & 32(26.2) \\ \text { Grade 3 } & (36.0) & & \\ & 54 & 14(21.9) & 40(32.8) \\ \text { Grade 4 } & (29.0) & & 43(35.2) \\ & 53 & 10(15.6) & \end{array}$

Tumor location, $\mathrm{N}(\%)$

0.594

Frontal

55

(29.6)

Temporal

44

(23.7)

Parietal

6 (3.2) $\quad 2$ (3.1)

$32(26.2)$

Occipital

2 (1.1) $0(0.0)$

29 (23.8)

Insula

$1(0.5)$

$0(0.0)$

2 (1.1) $1(1.6)$

$4(3.3)$

Thalamus

7 (3.8)

$0(0.0)$

$1(0.5) \quad 0(0.0)$

$2(1.6)$

Cerebellar hemisphere

Ventricle

3 (1.6) $1(1.6)$

$1(0.8)$

Basal ganglia

65
$(34.9)$

(34.9)

22 (34.4)

$1(0.8)$

Multiple lobes

Histopathological type, N (\%)

Glioblastoma, IDH-wild type

38

(20.4)

Diffuse astrocytoma, IDH-wild type

33

Anaplastic astrocytoma, IDHwild type

25

(13.4)

$4(6.3)$

7 (5.7)

$1(0.8)$

2 (1.6)

43 (35.2)

Diffuse astrocytoma, IDH-

$11(5.9) \quad 6(9.4)$

$5(4.1)$ 
mutant

Anaplastic oligodendroglioma, $9(4.8) \quad 1(1.6) \quad 8(6.6)$

NOS

Oligodendrocytoma, NOS

$9(4.8) \quad 7(10.9)$

$2(1.6)$

Astrocytoma, NOS

$8(4.3) \quad 4(6.3)$

4 (3.3)

Oligodendroglioma, NOS

$8(4.3) \quad 4(6.3)$

4 (3.3)

Anaplastic astrocytoma, NOS

$6(3.2) \quad 3(4.7)$

$53(2.5)$

Glioblastoma, NOS

$6(3.2) \quad 1(1.6)$

$5(4.1)$

Diffuse astrocytoma, NOS

$6(3.2) \quad 4(6.3)$

$2(1.6)$

Pilocytic astrocytoma

$5(2.7) \quad 0(0.0)$

$5(4.1)$

Gliosarcoma

4 (2.2) $0(0.0)$

4 (3.3)

Anaplastic astrocytoma, NOS

3 (1.6)

1 (1.6)

$2(1.6)$

Glioblastoma, IDH-mutant

$3(1.6) \quad 2(3.1)$

$1(0.8)$

Pilomyxoid astrocytoma

$2(1.1) \quad 1(1.6)$

$1(0.8)$

Gemistocytic astrocytoma, IDH- $1(0.5)$

wild type

$0(0.0)$

Gemistocytic astrocytoma, IDH- $1(0.5) \quad 0(0.0)$

$1(0.8)$

mutant

Anaplastic glioma, NOS

$1(0.5) \quad 0(0.0)$

$1(0.8)$

Anaplastic pleomorphic

xanthoastrocytoma

$1(0.5) \quad 0(0.0)$

$1(0.8)$

Anaplastic astrocytoma, IDHmutant

$1(0.5) \quad 0(0.0)$

$1(0.8)$

Glial hyperplasia

$1(0.5) \quad 1(1.6)$

$0(0.0)$ 


\begin{tabular}{|lccc|}
\hline Ganglioglioma & $1(0.5)$ & $1(1.6)$ & $0(0.0)$ \\
\hline Diffuse glioma, NOS & $1(0.5)$ & $0(0.0)$ & $1(0.8)$ \\
\hline Gangliocytoma & $1(0.5)$ & $0(0.0)$ & $1(0.8)$ \\
\hline Pilocytic astrocytoma & $1(0.5)$ & $1(1.6)$ & $0(0.0)$ \\
\hline
\end{tabular}

There was no statistical difference in tumor location between the epileptic group and the non-epileptic group $(P=0.594)$. Glioblastoma IDH wild-type and Anaplastic astrocytoma IDH wild-type were lower in the epileptic group, while diffuse astrocytoma IDH wild-type was higher in the epileptic group, and the difference of tumor histological type between the two groups was statistically significant $(P<0.001)$. The WHO pathological grade of epileptic group was mainly low grade, while that of non-epileptic group was mainly high grade, and the difference was statistically significant $(P<0.001)$.

\begin{tabular}{|c|c|c|c|c|c|c|c|}
\hline Variables & GFAP & Olig-2 & IDH-1 & ATR-X & Ki-67 & CD34 & Epilepsy \\
\hline GFAP & 1.00 & $0.25^{\star \star}$ & 0.05 & -0.10 & -0.12 & $0.16^{*}$ & 0.08 \\
\hline Olig-2 & & 1.00 & 0.01 & -0.03 & -0.07 & 0.13 & 0.03 \\
\hline IDH-1 & & & 1.00 & -0.03 & $-0.19^{\star}$ & -0.09 & $0.16^{*}$ \\
\hline ATR-X & & & & 1.00 & -0.004 & 0.04 & $-0.17^{*}$ \\
\hline Ki-67 & & & & & 1.00 & -0.07 & $-0.28^{\star \star}$ \\
\hline CD34 & & & & & & 1.00 & 0.03 \\
\hline Epilepsy & & & & & & & 1.00 \\
\hline \multicolumn{8}{|c|}{${ }^{*} p<0.05 ;^{* *} p<0.001}$. \\
\hline
\end{tabular}

Spearman correlation coefficient between idH-1 positive rate and tumor-related epilepsy was 0.16 , with a significant positive correlation $(P<0.05)$. Spearman correlation coefficient between the positive rate of ATR-X and ki-67 proliferation index and tumor-related epilepsy were -0.17 and -0.28 , respectively, and they were significantly negatively correlated with tumor-related epilepsy $(P<0.05)$ 


\begin{tabular}{|c|c|c|c|}
\hline Variables & & ORs (95\% Cls) & $P$-value \\
\hline \multirow[t]{2}{*}{ GPAF } & $(-)$ & 1.00 (reference) & - \\
\hline & $(+)$ & $0.31(0.02,5.09)$ & 0.409 \\
\hline \multirow[t]{2}{*}{ Oli-2 } & $(-)$ & 1.00 (reference) & - \\
\hline & $(+)$ & $1.14(0.32,4.07)$ & 0.840 \\
\hline \multirow[t]{2}{*}{$\mathrm{IDH}-1$} & $(-)$ & 1.00 (reference) & - \\
\hline & $(+)$ & $2.30(0.94,5.63)$ & 0.068 \\
\hline \multirow[t]{2}{*}{ ATR-X } & $(-)$ & 1.00 (reference) & - \\
\hline & $(+)$ & $0.44(0.21,0.92)$ & 0.029 \\
\hline \multirow[t]{3}{*}{ Ki-67 } & Low & 1.00 (reference) & - \\
\hline & Medium & $0.67(0.27,1.68)$ & 0.394 \\
\hline & High & $0.25(0.10,0.68)$ & 0.006 \\
\hline \multirow[t]{2}{*}{ CD34 } & $(-)$ & 1.00 (reference) & - \\
\hline & $(+)$ & $0.86(0.38,1.96)$ & 0.718 \\
\hline
\end{tabular}

Multiple logistic regression was used to analyze the association between immunohistochemical indexes and the risk of glioma complicated with epilepsy, and the results were shown in Table 4. After adjusting for gender, age, and WHO pathological grade, the incidence of epilepsy in patients with ATR-x positive glioma was significantly reduced $(P=0.029)$ and the risk was $56 \%$ lower $(O R=0.44)$ than in the ATR-X negative group. $95 \% \mathrm{Cl}: 0.21,0.92)$; Compared with the low-proliferation ki-67 group, the incidence of epilepsy in glioma patients in the high-proliferation $\mathrm{KI}-67$ group was significantly reduced $(\mathrm{P}=0.006)$, and the risk was reduced by $75 \%(\mathrm{OR}=0.25 ; 95 \% \mathrm{Cl}: 0.10,0.68)$.

\section{Figures}



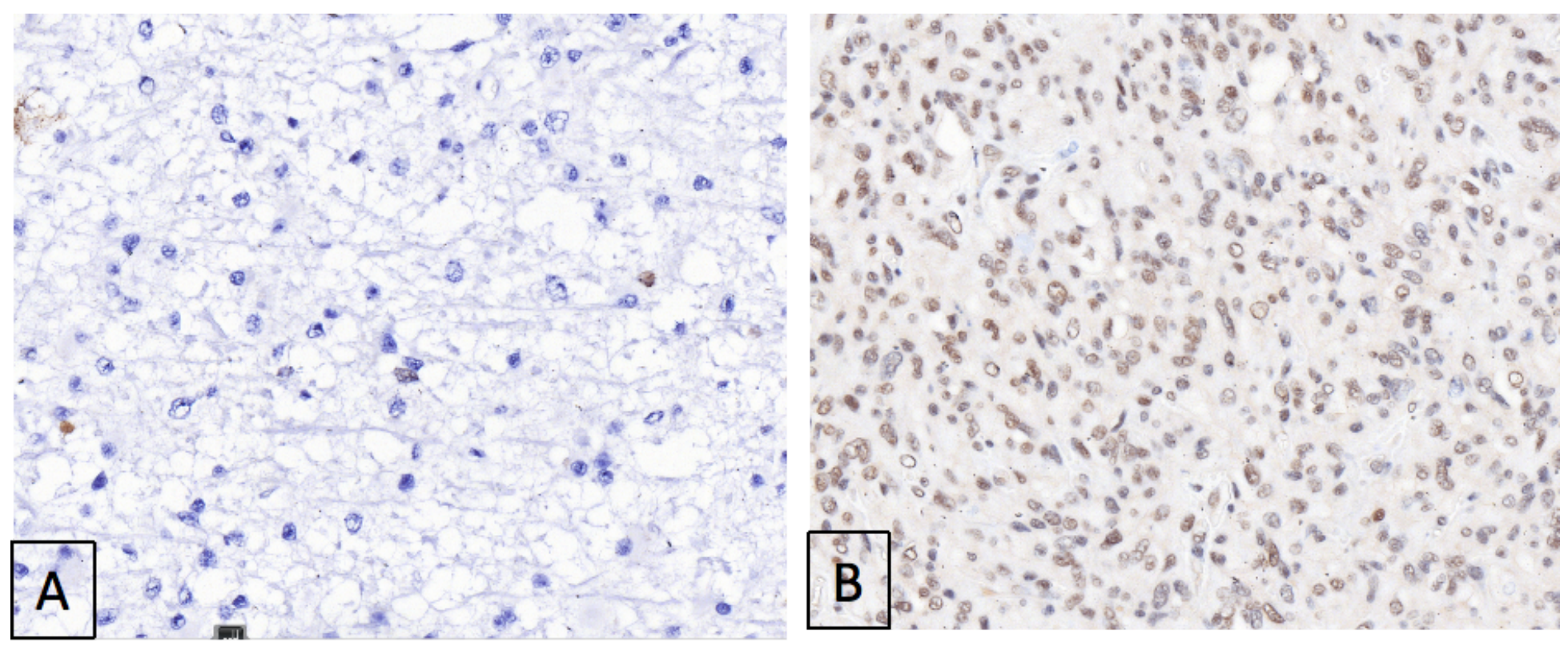

Figure 1

Photomicrographs of tumor tissue stained for ATR-X: (A) negative expression, (B) positive expression, original magnification: $\mathrm{x} 400$.

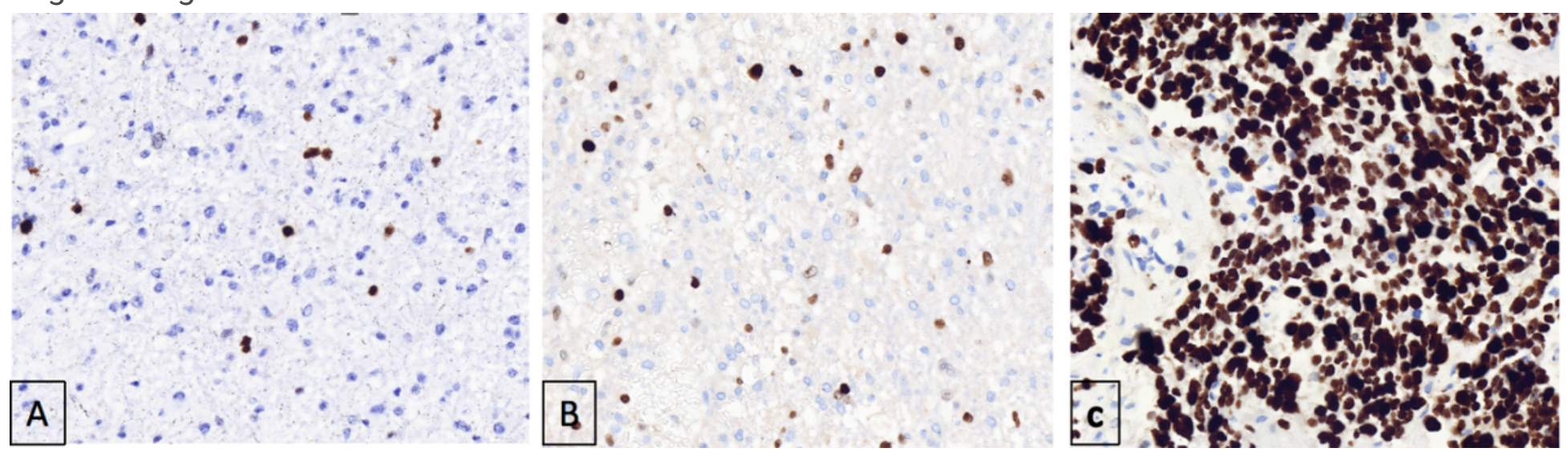

Figure 2

Photomicrographs of tumor tissue stained for ki67 PI: (A) low ki67 PI ( $\leq 5 \%)$, (B) medium ki67 PI (6-20\%), (C) high ki67 PI (>20\%), original magnification: x400. 


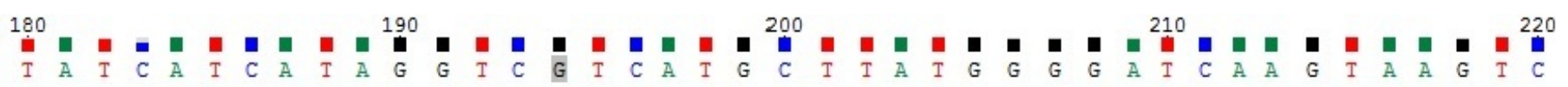
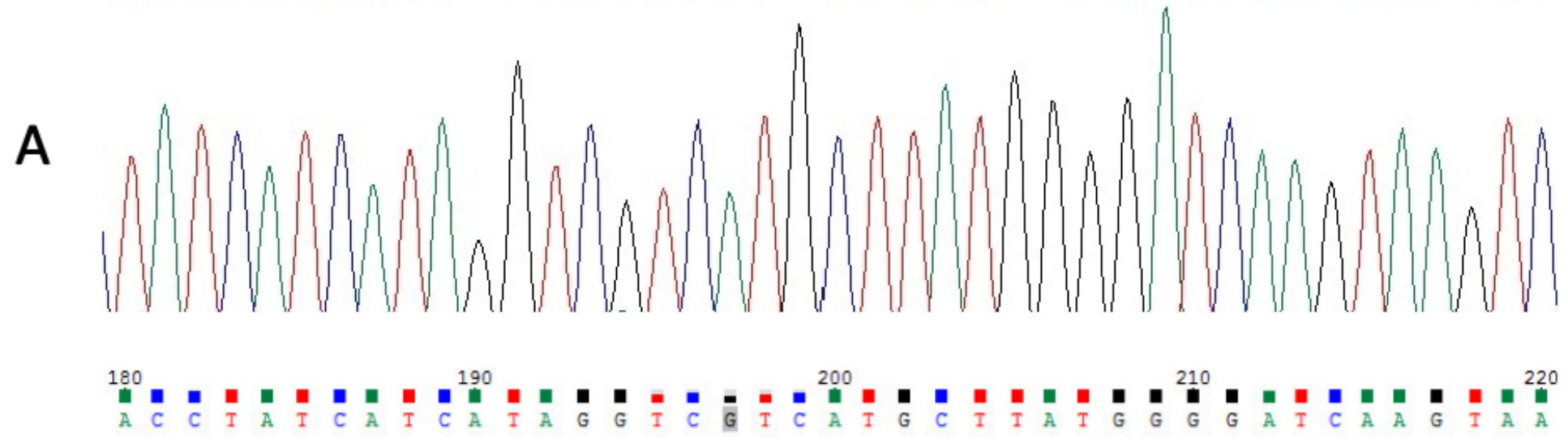

B

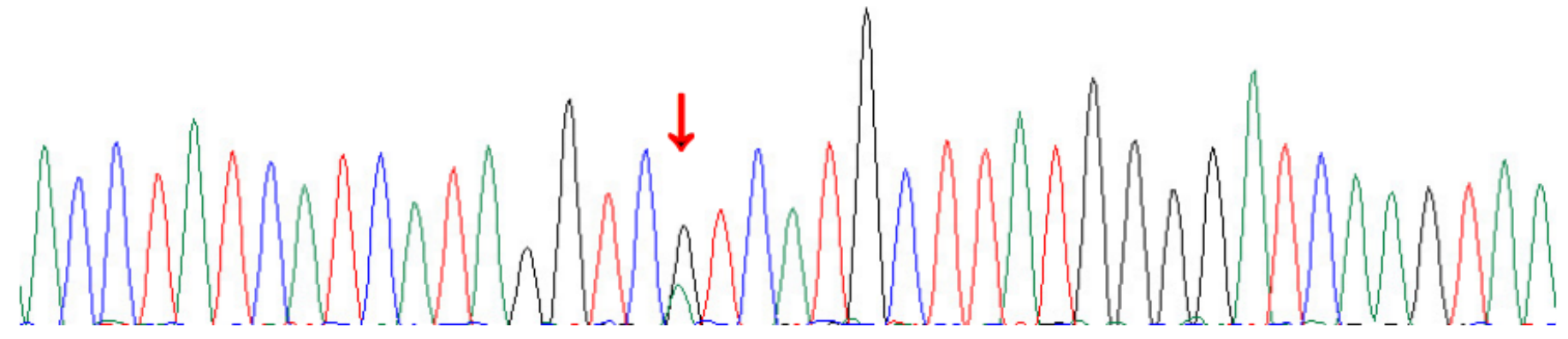

Figure 3

Sanger sequencing analysis of IDH1 R132H mutation with negative (A) and positive (B)(the red arrow) results. 